\title{
CARACTERÍSTICAS COLORIMÉTRICAS ENTRE AS SEÇÕES ANATÔMICAS DA MADEIRA DE MUIRAPIRANGA (Brosimum sp.)
}

\author{
Claudene Menezes Atayde ${ }^{1}$, Joaquim Carlos Gonçalez², José Arlete Camargos ${ }^{3}$
}

(recebido: 2 de outubro de 2009; aceito: 25 de fevereiro de 2011)

\begin{abstract}
RESUMO: Objetivou-se, com este trabalho, avaliar as características colorimétricas das três seções anatômicas (transversal, tangencial e radial) da madeira de muirapiranga (Brosimum sp.), utilizando o sistema CIELAB 1976 (Comissão Internacional de Iluminantes). A partir de 10 amostras de madeira, obtidas em movelarias do município de Cruzeiro do Sul - AC, foram determinados os parâmetros colorimétricos: luminosidade $\left(\mathrm{L}^{*}\right)$, coordenada do eixo vermelho-verde $\left(\mathrm{a}^{*}\right)$, coordenada do eixo azul-amarelo (b*), saturação (C) e ângulo de tinta (h*). Foram tomadas em média 34 medições por corpo-de-prova, em cada um dos planos ou seções. Dessa forma foram obtidos os seguintes parâmetros colorimétricos para a espécie Brosimum sp.: L*=42,13, a*=18,87, b*=15,05, $\mathrm{C}=24,23$ e $\mathrm{h}^{*}=37,86$. Houve variações de cor, mostrando diferenças significativas entre as três seções estudadas. Em relação à reflectância, observou-se que a seção transversal (6-28\%) refletiu menos luz que as seções tangencial (6-43\%) e radial (7-49\%). Conforme o plano anatômico da madeira, pode-se obter madeiras com diferentes tons de vermelho, permitindo a formação de lotes de madeira com a mesma tonalidade.
\end{abstract}

Palavras-chave: Madeira da Amazônia, CIELAB, indústria moveleira.

\section{COLORIMETRIC CHARACTERISTICS OF DIFFERENT ANATOMICAL SECTIONS OF MUIRAPIRANGA (Brosimum sp.) WOOD}

\begin{abstract}
This work aimed to evaluate and compare colorimetric characteristics of three anatomical sections (transverse, tangential and radial) of 'muirapiranga'bloodwood (Brosimum sp.), by using the International Commission on Illumination system, CIELAB 1976 (Comissão Internacional de Iluminantes). By using 10 samples obtained from furniture traders in the municipality of Cruzeiro do Sul (AC), the following colorimetric parameters were determined: lightness $(L *)$, red-green axis ( $\left.a^{*}\right)$, blue-yellow axis $\left(b^{*}\right)$, chrome $(C)$ and hue angle $\left(h^{*}\right)$. An average 34 readings were taken per specimen in each of the planes or sections. The following colorimetric parameters were derived for Brosimum sp. species: $L^{*}=42.13, a^{*}=18.87, b^{*}=15.05, C=24.23$ and $h *=37.86$. There were variations in color, indicating significant differences among the three sections in question. As to reflectance, the transverse section (6-28\%) was found to reflect less light than the tangential (6-43\%) and radial sections (7-49\%). Depending on which anatomical plane is chosen, different shades of red can be obtained, allowing forming similarly shaded wood batches.
\end{abstract}

Key words: Amazonia wood, CIELAB, furniture industry.

\section{INTRODUÇÃO}

A madeira, por sua natureza biológica, é bastante heterogênea apresentando as mais diversas características resultantes de sua composição química e anatômica. Essa combinação durante o desenvolvimento da planta resultará em uma grande variabilidade de cores apresentadas na madeira formada. Essas cores dependem dos componentes químicos da madeira que interagem com a luz (HON; MINEMURA, 2001).

Os componentes orgânicos da madeira são, principalmente, os polissacarídeos e os polifenóis: celulose, hemicelulose e lignina. Os extrativos também estão presentes, contudo em quantidades relativamente menores e sua concentração determina a cor, o odor e outras propriedades não-mecânicas da madeira (FEIST; HON, 1984; PANSHIN; ZEEUW, 1970; UMEZAWA, 2001). Devido às diferenças na composição destes componentes, a cor da madeira não tratada varia entre diferentes espécies, entre diferentes árvores da mesma espécie e, até mesmo, dentro da mesma árvore (MÖTTÖNEN, 2005; NISHINO et al., 1998).

A mudança da cor da madeira está relacionada à decomposição da lignina nas células da superfície da madeira, um fenômeno que ocorre apenas na superfície (0,05-2,5mm), e é resultado da incidência da luz ultravioleta que inicia a fotodegradação (FEIST; HON, 1984).

\footnotetext{
${ }^{1}$ Engenheira Florestal, Doutoranda em Ciências Florestais na Universidade de Brasília, Professora da Universidade Federal do Acre - Cx. P. 4336 70904-970 - Brasília, DF - claudeneatayde@hotmail.com

${ }^{2}$ Engenheiro Florestal, Professor Dr. em Ciências Florestais - Departamento de Engenharia Florestal - Universidade de Brasília - 70919 -970 Brasília, DF - goncalez@unb.br

${ }^{3}$ Economista, Mestre em Ciências Florestais e Ambientais - Instituto Brasileiro do Meio Ambiente e dos Recursos Naturais Renováveis/IBAMA Laboratório de Produtos Florestais - Brasília, DF - camargos@lpf.ibama.gov.br.
} 
Com a crescente demanda do mercado na busca de um produto de melhor qualidade, surgiu a necessidade de medição do estado de superfície para alguns tipos de materiais, dentre estes a madeira. Neste contexto, a colorimetria se apresenta como uma das metodologias para a análise de superfícies de madeira (NISHINO et al., 1998; SIQUEIRA et al., 2003).

A colorimetria descreve cada elemento da composição de uma cor, numericamente, por meio de aparelhos apropriados. Esta metodologia de análise de cor é usada em diferentes sistemas de produção fabril, como, por exemplo, indústrias têxteis, automotivas, químicas e plásticas. Um dos sistemas mais utilizados para medição de cores é o CIELAB (Comissão Internacional de Iluminantes), que é sintetizado através dos parâmetros colorimétricos: luminosidade $\left(\mathrm{L}^{*}\right)$, coordenada do eixo vermelho-verde $\left(\mathrm{a}^{*}\right)$, coordenada do eixo azul-amarelo (b*), saturação (C) e ângulo de tinta (h*) (CAMARGOS; GONÇALEZ, 2001).

Camargos e Gonçalez (2001) relatam que a cor é uma das características mais importantes para a identificação e indicação de usos de espécies de madeira, principalmente quando associada aos aspectos de textura e desenho. A cor pode ser alterada pelo teor de umidade, pela temperatura, pelas degradações provocadas pelo ataque de organismos xilófagos ou, ainda, pelas reações fotoquímicas dos componentes químicos presentes em sua estrutura. A idade da madeira e da árvore também são fatores que influenciam a cor natural deste material, assim como os tratos silviculturais e o tipo de solo onde se desenvolveu a planta (SOTELO MONTES et al., 2008).

A homogeneidade da cor da madeira em um lote a ser utilizado industrialmente é um quesito básico de qualidade, uma vez que refletirá na homogeneidade dos produtos resultantes de seu beneficiamento. Com isso, torna-se clara a necessidade da determinação da cor da madeira e sua variabilidade como instrumento de tomada de decisão sobre a sua utilização como matéria-prima de um segmento industrial que deve sempre atentar para o valor estético de seus produtos (ABRAHÃO, 2005).

A análise final da cor de qualquer produto lhe garante um padrão de qualidade de mercado exigido mundialmente. Ela é um dos componentes da estética, que se associa à superfície e ao desenho de uma peça de madeira. Por essa razão, esta propriedade deve ser incorporada ao planejamento visando à caracterização tecnológica da madeira, para atender aos usos mais nobres deste material (MORI et al., 2005).

Cerne, Lavras, v. 17, n. 2, p. 231-235, abr./jun. 2011
Assim, os estudos sobre espécies de madeira alternativas devem ter como princípios as propriedades tecnológicas como base científica para definição da qualidade da matéria-prima.

Brosimum sp., família Moraceae, é uma espécie arbórea, de altura entre 30 e 50m, com ampla distribuição na Amazônia. É conhecida popularmente como muirapiranga e pau-rainha (LOUREIRO et al., 1997) e utilizada na fabricação de móveis de luxo, instrumentos musicais, objetos de adorno, entre outros. A madeira apresenta coloração avermelhada intensa (um dos principais atrativos), tronco reto e longo, é resistente ao ataque de fungos e insetos, possui boa trabalhabilidade e tem pouca propensão as rachaduras e empenos (SOUZA, 1998).

No município de Cruzeiro do Sul - AC, pode-se observar, por meio de visitas em movelarias, que a madeira de muirapiranga é bastante utilizada na fabricação de móveis, tendo boa aceitação no mercado local.

Portanto, objetivou-se, com este estudo, caracterizar a cor das seções transversal, tangencial e radial, da madeira de muirapiranga (Brosimum sp.) utilizando-se o sistema CIELAB 1976.

\section{MATERIAL E MÉTODOS}

\subsection{Preparação das amostras}

As amostras da madeira de muirapiranga utilizadas foram coletadas em três movelarias, no município de Cruzeiro do Sul - AC. Em duas empresas, foram obtidas três pranchas, e, na terceira, de maior porte, quatro pranchas, totalizando dez peças. Medindo em média 4m x 0,30m x 0,040m (comprimento, largura e espessura), as dez pranchas foram desdobradas em amostras orientadas medindo $2 \mathrm{~cm} \times 2 \mathrm{~cm} \times 10 \mathrm{~cm}$, totalizando 10 corpos-deprova, os quais foram lixados antes da análise colorimétrica. Cada uma das amostras foi anatomicamente identificada no setor de Anatomia da Madeira do Laboratório de Produtos Florestais (LPF/SBF).

\subsection{Medição da cor}

Para determinação da cor da madeira utilizou-se o método CIELAB 1976 com o uso do espectrofotômetro Datacolor International Microflash - Model 200, acoplado a um computador. O iluminante utilizado foi o D 65, lâmpada de xenônio que simula a radiação solar diurna, recomendado para o uso em colorimetria e ângulo do observador de $10^{\circ}$. Esses dados foram coletados no Laboratório de Produtos Florestais - LPF/SFB. 
A medição da cor foi feita nas seções transversal, tangencial e radial, em cada uma das amostras, sendo realizadas 34 medições em cada uma das seções, totalizando uma média de 340 medições por seção estudada. Os parâmetros colorimétricos obtidos foram os seguintes: $\mathrm{L}^{*}$ (luminosidade), a* (coordenada do eixo vermelho-verde), b* (coordenada do eixo azul-amarelo).

Para o cálculo dos parâmetros C (saturação) e h* (ângulo de tinta), foram utilizadas as equações (1) e (2), conforme procedimento descrito por Gonçalez (1993).

$$
\begin{aligned}
& C=\frac{\left(a^{* 2}+b^{* 2}\right)}{2} \\
& h^{*}=\tan ^{-1}\left(\frac{b^{*}}{a^{*}}\right)
\end{aligned}
$$

\subsection{Análise Estatística}

Os valores obtidos dos parâmetros colorimétricos foram comparados estatisticamente pelo teste $D$ de Duncan para comparação de médias a 5\% de probabilidade.

\section{RESULTADOS E DISCUSSÃO}

A Tabela 1 apresenta os parâmetros colorimétricos médios das seções transversal, tangencial e radial.

Tabela 1 - Parâmetros colorimétricos da madeira de Brosimum sp.

Table 1 - Colorimetric parameters of Brosimum sp wood.

\begin{tabular}{lccccc}
\hline \multirow{2}{*}{ Planos } & \multicolumn{5}{c}{ Parâmetros colorimétricos } \\
\cline { 2 - 6 } & $\mathrm{L}^{*}$ & $\mathrm{a}^{*}$ & $\mathrm{~b}^{*}$ & $\mathrm{C}$ & $\mathrm{h}^{*}$ \\
\hline Transversal & $35,60^{\mathrm{a}}$ & $14,65^{\mathrm{a}}$ & $9,67 \mathrm{a}$ & $17,59 \mathrm{a}$ & $33,12 \mathrm{a}$ \\
Tangencial & $42,85 \mathrm{~b}$ & $20,69 \mathrm{~b}$ & $16,39 \mathrm{~b}$ & $26,45 \mathrm{~b}$ & $38,36 \mathrm{~b}$ \\
Radial & $47,93 \mathrm{c}$ & $21,26 \mathrm{~b}$ & $19,09 \mathrm{c}$ & $28,66 \mathrm{c}$ & $42,09 \mathrm{c}$ \\
Média geral & 42,13 & 18,87 & 15,05 & 24,23 & 37,86 \\
\hline
\end{tabular}

Média aritmética de 340 medições para cada uma das seções. Médias seguidas pela mesma letra, dentro da mesma coluna, não se diferenciam estatisticamente ao nível de 5\% de probabilidade pelo teste de Duncan.

Analisando-se a Tabela 1, verificou-se que o valor da luminosidade $\left(L^{*}\right)$ foi menor na seção transversal em relação aos outros dois planos. No trabalho de Nishino et al. (2000), também foi observado comportamento semelhante, que a seção transversal das células de fibra pode diminuir a luminosidade, não por causa dos lumens, e sim devido à luminosidade de substâncias presentes nas paredes das células e características anatômicas de cada plano. Por outro lado, Gonçalez et al. (2001) relatam que, na seção transversal, a presença dos anéis de crescimento aumenta o nível de cinza, fazendo com que haja uma diminuição da luminosidade ( $\left.L^{*}\right)$ e dos matizes responsáveis pelas coordenadas a* e b*, tornando a madeira mais escura.

As três seções anatômicas são influenciadas pelas coordenadas cromáticas a* e b*, as quais caracterizam a cor da madeira. Observa-se que os valores das duas coordenadas na seção transversal são menores, quando comparados ao das seções tangencial e radial. Alguns espécimes têm diferença de cor e aparência entre as seções tangencial e radial as quais podem ser atribuídas às características anatômicas (NISHINO et al., 1998).

O ângulo de tinta ou tonalidade também apresentou menor valor na seção transversal, em relação às outras duas, indicando que a transversal é mais escura que as demais. O parâmetro saturação ou cromaticidade (C) também é influenciado pelas coordenadas a* e b*, dessa forma à medida que estas duas variáveis aumentam os valores da saturação também aumentam. O aumento desse valor de cromaticidade evidencia um aumento nos pigmentos vermelho e amarelo, indicando maior saturação da cor. Verifica-se que a seção radial é a que apresenta a coloração mais saturada e a maior luminosidade. Segundo Möttönen (2005), estudos microscópicos revelaram que produtos químicos descoloridos na madeira estão concentrados nas células de parênquima dos raios, o que poderia explicar o fato de a seção radial apresentar coloração mais clara, devido a maior proximidade entre as células radiais.

De acordo com Gonçalez et al. (2001), a coordenada $a^{*}$, quando positiva e crescente, significa presença da coloração avermelhada mais pronunciada e quando a coordenada b* aumenta, significa a presença da cor amarela. Nesta pesquisa, a coordenada $a^{*}$, que representa predominância do pigmento vermelho, é marcante na espécie muirapiranga.

Neste trabalho foram encontrados valores médios dos parâmetros analisados (Tabela 1) próximos aos observados por Autran e Gonçalez (2006) que encontraram para a madeira de muirapiranga os seguintes valores: $L^{*}=42,39 ; a^{*}=22,02 ; b^{*}=19,61 ; C=26,99$ e $h^{*}=35,10$.

Cerne, Lavras, v. 17, n. 2, p. 231-235, abr./jun. 2011 
Observa-se ainda, na Tabela 1 que, com exceção da coordenada a*, entre as seções tangencial e radial, todos os valores dos parâmetros analisados entre os três planos da madeira apresentaram entre si diferenças significativas pelo teste de Duncan a 5\% de probabilidade. Este resultado foi diferente do encontrado por Autran e Gonçalez (2006), pois estes autores não encontraram diferenças significativas entre as seções tangencial e radial da madeira de muirapiranga proveniente do Estado de Mato Grosso. As condições dos sítios podem ter influenciado no desenvolvimento da planta, na qualidade da madeira e em suas propriedades. Seria aconselhável um estudo para avaliar a influência do ambiente nas características colorimétricas da madeira.

A seção transversal apresentou maior valor de pigmentação vermelha em relação às outras duas, porém com menor valor de luminosidade, refletindo uma coloração mais escura nessa seção de desdobro.

Entre as seções tangencial e radial, a coordenada a* não apresentou diferenças significativas. Segundo Autran e Gonçalez (2006), a pigmentação amarela presente nesta espécie é decisiva para a formação da sua cor, podendo-se esperar que a madeira de muirapiranga apresente nuances de pigmentos avermelhados mais vivos na direção radial de desdobro, o que pode ser confirmado, segundo os autores, pelo parâmetro C, o qual varia proporcionalmente aos valores de $a^{*}$ e b*. Comportamento semelhante foi encontrado neste trabalho.

A Figura 1 mostra a curva de reflectância da madeira de Brosimum sp. em função do comprimento de onda, nas seções transversal, tangencial e radial.

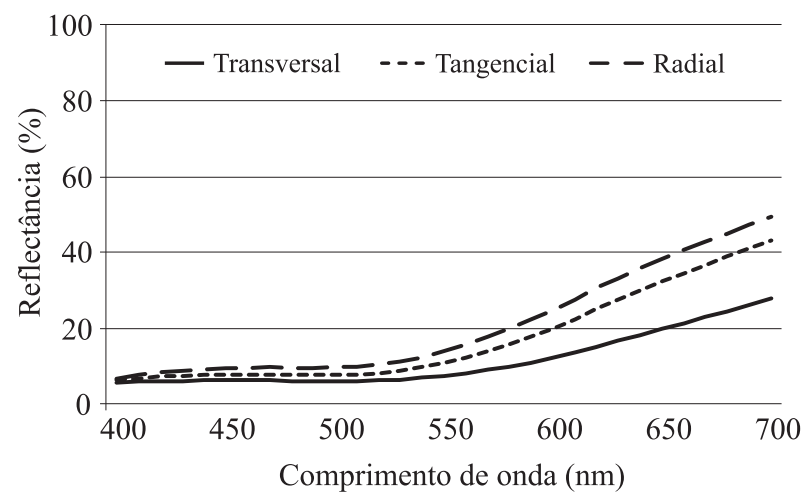

Figura 1 - Curva de reflectância da madeira de muirapiranga (Brosimum sp.), em função do comprimento de onda, nas seções transversal, tangencial e radial.

Figure 1 - Reflectance curve of muirapiranga wood (Brosimum sp.) as a function of wavelength on transverse, tangential and radial sections.

Cerne, Lavras, v. 17, n. 2, p. 231-235, abr./jun. 2011
Os valores de reflectância nas seções estudadas apresentaram valores entre 6 e 49\%, sendo que essa porcentagem se apresentou da seguinte forma: na seção transversal, variou de 6 a 28\%, na tangencial, de 6 a $43 \%$ e, na radial, de 7 a 49\%. Esses valores variaram conforme o valor de L* aumentava: 35,60 na seção transversal, 42,85 na seção tangencial e 47,93 na seção radial. Isso é, a curva para a seção transversal reflete menos luz que as outras duas seções. Isso pode ser explicado pelo menor valor da claridade nesta seção. Observa-se ainda que, para as três seções, os comprimentos de onda de maior reflectância estão na faixa 580 a 700nm (nanômetros). Justamente a faixa das cores amarelo-laranja-vermelho. Entre as três seções nesta faixa, a radial é a que tem maior reflectância e a transversal a menor, confirmando a maior e a menor claridade destas seções.

\section{CONCLUSÕES}

A madeira de muirapiranga apresentou diferenças de cor entre as seções anatômicas estudadas (transversal, tangencial e radial), sendo caracterizada pela coloração vermelha, a qual é definida pelo alto valor do parâmetro a* (pigmentação vermelha).

O uso do sistema CIELAB 1976 permitiu caracterizar e diferenciar de forma eficaz as tonalidades entre as seções anatômicas estudadas da madeira de muirapiranga, detectado pelos parâmetros colorimétricos $\left(L^{*}, a^{*}, b^{*}, C, h^{*}\right)$.

Conforme a forma de desdobro da tora, se pode obter madeiras mais avermelhadas (corte tangencial) ou com tons mais amarelo-avermelhado (corte radial), permitindo a formação de lotes de madeira com tonalidades mais homogêneas, de acordo com as preferências de mercado.

\section{REFERÊNCIAS}

ABRAHÃO, C. P. Estimativa de algumas propriedades da madeira de Eucalyptus urophylla por espectrometria. 2005. 182 p. Tese (Doutorado em Ciência Florestal) - Universidade Federal de Viçosa, Viçosa, 2005.

AUTRAN, C. S.; GONÇALEZ, J. C. Caracterização colorimétrica das madeiras de muirapiranga (Brosimum rubescens Taub.) e de seringueira (Hevea brasiliensis, clone Tjir 16 Müll Arg.) visando à utilização em interiores. Ciência Florestal, Santa Maria, v. 16, n. 4, p. 445-451, 2006. 
CAMARGOS, J. A. A.; GONÇALEZ, J. C. A colorimetria aplicada como instrumento na elaboração de uma tabela de cores de madeira. Brasil Florestal, Brasília, n. 71, p. 30-41, set. 2001.

FEIST, C. W.; HON, D. S. Chemistry of weathering and protection. In: ROWELL, R. M. The chemistry of solid wood: advances in chemistry series 207. Washington: American Chemical Society, 1984. chap. 11.

GONÇALEZ, J. C. Caracterisation technologique de quatre espéces peu connues de la forêt Amazonienne: anatomie, chimie, couleur, propriétés physiques et mécaniques. 1993. 446 p. Thèse (Doctorat) - ENGREF, Nancy, 1993.

GONÇALEZ, J. C.; JANIN, G.; SANTORO, A. C. S.; COSTA, A. F.; VALLE, A. T. Colorimetria quantitativa: uma técnica objetiva de determinar a cor da madeira. Brasil Florestal, ano 20, n. 72, p. 47-58, 2001.

HON, D. N. S.; MINEMURA, N. Color and discoloration. In: HON, D. N. S.; SHIRAISHI, N. (Eds.). Wood and cellulosic chemistry. 2. ed. rev. and exp. New York: M. Dekker, 2001. p. 385-442.

LOUREIRO, A. A.; FREITAS, J. A.; FREITAS, C. A. A. Essências madeireiras da Amazônia. Manaus: MCT/INPACPPF, 1997. 89 p.

MORI, C. L. S. O.; LIMA, J. T.; MORI, F. A.; TRIGILHO, P. F.; GONÇALEZ, J. C. Caracterização da cor da madeira de clones de híbridos de Eucalyptus spp. Cerne, Lavras, v. 11, n. 2, p. 137-146, abr./jun. 2005.

MÖTTÖNEN, V. Variation of color and selected physical and mechanical properties related to artificial drying of sawn silver birch (Betula pendula Benth) timber from plantations. 2005. 43 p. Dissertationes (Mesters in Forestales) - Faculty of Forestry, University of Joensuu, 2005.

NISHINO, Y.; JANIN, G.; CHANSON, B.; DÉTIENNE, P.; GRIL, J.; THIBAUT, B. Colorimetry of wood specimens from French Guiana. Journal Wood Science, v. 44, p. 3-8, 1998.

NISHINO, Y.; JANIN, G.; YAMADA, Y.; KITANO, D. Relations between the colorimetric values and densities of sapwood. Journal Wood Science, v. 46, p. 267-272, 2000.

PANSHIN, A. J.; ZEEUW, C. Textbook of wood technology. 3. ed. New York: McGraw-Hill, 1970. v. 1.

SIQUEIRA, K. P.; MONTEFUSCO, A. R. G.; GUIMARÃES NETO, A. B.; BONDUELLE, A. Principais metodologias de medição de estados de superfície. Revista Ciências Exatas e Naturais, v. 5, n. 2, jul./dez. 2003.

SOTELO MONTES, C.; HERNANDÉZ, R. E.; BEAULIEU, J.; WEBER, J. C. Genetic variation in wood color and its correlations with tree growth and wood density of Calycophyllum spruceanum at an early age in Peruvian Amazon. New Forests, v. 35, p. 57-73, 2008.

SOUZA, M. H. Incentivo ao uso de novas madeiras para fabricação de móveis. 2. ed. Brasília: IBAMA, 1998. 70 p.

UMEZAWA, T. Chemistry of extractives. In: HON, D. N. S.; SHIRAISHI, N. (Eds.). Wood and cellulosic chemistry. 2. ed. rev. and exp. New York: M. Dekker, 2001. p. 213242.

Cerne, Lavras, v. 17, n. 2, p. 231-235, abr./jun. 2011 